\title{
Kein Eigentumsverlust an NS-verfolgungsbedingt veräußertem Gemälde
}

\author{
Landgericht Berlin, Urteil vom 31. Januar 2008 - 27 O 89/07
}

\section{Ein im April 1935 in Berlin NS-verfolgungsbedingt veräußertes Gemälde kann im Sinne des § 935 Abs. 2 BGB abhanden gekommen sein.}

2. Die Washingtoner Prinzipien und die sog. Gemeinsame Erklärung haben keinen Einfluss auf privatrechtliche Rechtspositionen außerhalb der öffentlichen Hand.

3. Denjenigen, der sich auf einen gutgläubigen Erwerb außerhalb der Sphäre des ursprünglichen Eigentümers beruft, trifft im Rahmen des § 138 Abs. 1 ZPO trotz der Darlegungs- und Beweislastverteilung des §937 BGB eine erweiterte Darlegungslast hinsichtlich seiner Gutgläubigkeit für Erwerbsvorgänge in seiner Sphäre. (Leitsätze der Redaktion)

\section{Tatbestand}

- Der Kläger begehrt die Feststellung seines Eigentums an einem Gemälde sowie die Streichung von Einträgen hinsichtlich dieses Gemäldes aus Datenbanken.

Die Beklagte war Eigentümer des Gemäldes „A." von B. Am 26. oder 27. April 1935 wurden die Bestände der Beklagten, $u$. a. das hier streitgegenständliche Gemälde, im Auktionshaus C. versteigert. Auf der Titelseite des seinerzeitigen Katalogs hieß es, dass sich die Beklagte „in Liquidation“ befinde. In einer Vereinbarung zwischen der Bundesrepublik Deutschland und der Beklagten hinsichtlich eines anderen im Auktionshaus $C$. am 3. und 4. Mai 1935 veräußerten Bildes heißt es, dass die seinerzeitigen Gesellschafter der Beklagten, die Eheleute D., als Juden Verfolgungsmaßnahmen der Nationalsozialisten ausgesetzt gewesen seien und sich gezwungen gesehen hätten, den Geschäftsbetrieb der Beklagten aufzugeben und zu emigrieren. Die Veräußerung des Gemäldes im Rahmen der Versteigerung sei als verfolgungsbedingter Vermögensverlust im Sinne des Art. 3 der BK/O (49) 180 [REAO] anzusehen. Dieses andere Gemälde wurde daher der Beklagten von der Bundesrepublik zurückgegeben.

Bei der Versteigerung am 26./27. April 1935 erwarb das hier streitgegenständliche Gemälde E., dessen Vermögen von der Gestapo beschlagnahmt wurde. Über den weiteren Verbleib des Gemäldes ist nichts bekannt. Erst für eine Versteigerung am 8. Mai 1969 wurde das Gemälde durch das Kunsthaus F. als Los Nummer G. im Katalog angeboten. Es war ein Preis von [...] DM verzeichnet. Laut dem Katalog lag ein „Gutachten M. J. Friedländer" vor. Die Versteigerung am 8. Mai 1969 wurde durch H. durchgeführt. Ob das Gemälde aber bei dieser Versteigerung tatsächlich versteigert wurde, ist zwischen den Parteien streitig. Im Jahr 1989 wurde das Bild auf einer Versteigerung von I. in New York angeboten. Im dortigen Katalog hieß es unter Herkunft: „Probably J., Berlin before 1932". 1993 versuchte K., in dessen Besitz das Gemälde zwischenzeitlich gelangt war, erfolglos, das Bild bei L. in München zu versteigern. Im dortigen Katalog war als Voreigentümer die Beklagte benannt. Danach bot er das Gemälde 1997 in Spanien ohne Erfolg zum Verkauf an. Danach gelangte es in den Besitz des Klägers, der es 2002 bei der Galerie M. einlieferte, um es zu veräußern, was sich bisher als nicht möglich erwies, weil das Gemälde im Internet als Raub- und Beutekunst aufgelistet ist, nämlich einerseits auf der Internetseite der „Koordinierungsstelle für Kulturgutverluste" in Magdeburg unter "lostart.de" sowie andererseits auf der Internetseite des Londoner "Art Loss Register" unter "artloss.com”. Am 2. Dezember 2002 erkundigte sich das Art Loss Register bei der vom Kläger beauftragten Galerie nach dem Gemälde. Das Art Loss Register und die Koordinierungsstelle löschten auf Bitten der Galerie die Einträge nicht, da dort die Zustimmung der Beklagten fehle. Die Beklagte verweigerte eine solche Zustimmung.

Nachdem der Kläger in der Klageschrift vorbringen ließ, das Gemälde sei bei der Beklagten im Jahr 1935 beschlagnahmt worden und daher abhanden gekommen, bestreitet er dies nun. Die Eheleute D. seien schon zu Beginn der nationalsozialistischen Herrschaft nicht mehr Eigentümer des Unternehmens gewesen. Der Kläger behauptet, $\mathrm{H}$. sei öffentlich bestellter und vereidigter Versteigerer gewesen und als solcher im Rahmen der Versteigerung vom 8. Mai 1969 tätig geworden. Das Gemälde sei als Los Nr. G. für [...] DM versteigert worden. Der Katalog bei der Versteigerung im Jahr 1969 verweise lediglich hinsichtlich des Zustands des Gemäldes auf das Gutachten des Hr. Friedländer. Am 13. Oktober 1989 habe K., der Schwiegervater des Klägers, das Gemälde auf einer Versteigerung in New York beim Auktionshaus I. für [...] US-Dollar ersteigert. Er, der Kläger, habe das Gemälde im Jahr 1997 von K. gekauft. Der Kläger meint, er sei Eigentümer des Gemäldes. Es sei 1969 bei der Versteigerung des Kunsthauses F. vom Ersteigerer gutgläubig erworben worden gemäß § 935 Abs. 2 BGB. Anhaltspunkte dafür, dass dem Versteigerer oder dem Ersteigerer die Herkunft des Bildes bekannt gewesen wären, seien nicht ersichtlich und zudem von der Beklagten darzulegen und zu beweisen. Bei den nachfolgenden Veräußerungen sei jeweils Eigentum nach § 929 S. 1 BGB übertragen worden.

Jedenfalls habe er das Gemälde ersessen gemäß §§ 937, 943 BGB, da die 10-jährige Ersitzungszeit seit dem Oktober 1989 abgelaufen sei. Im Übrigen sei ein etwaiger Herausgabeanspruch der Beklagten verjährt.

Die Eintragungen auf den genannten Internetseiten seien zu löschen, weil das Bild dadurch praktisch unverkäuflich sei. 
Ziel der Datenbanken sei es, nach verschwundenen Bildern zu suchen und eine Eigentumsklärung zu ermöglichen. Diese Voraussetzungen lägen nicht vor.

Der Kläger beantragt,

1. festzustellen, dass er Eigentümer des Gemäldes „A." von B. ist;

2. die Beklagte zu verurteilen, durch Erklärung gegenüber

a) der Koordinierungsstelle für Kulturgutverluste, Turmschanzenstraße 32, 39114 Magdeburg,

b) "The Art Loss Register", First Floor, 63 - 66 Harton Garden, GB London EC1N 8LE, England,

einer Löschung des Gemäldeeintrags „A.“ von einer Liste gesuchter Raub- und Beutekunst auf der Internetseite www.lostart.de zuzustimmen.

\section{Die Beklagte beantragt, die Klage abzuweisen.}

Sie behauptet, das Bild sei ihr 1935 als Folge nationalsozialistischer Verfolgung abhanden gekommen. Sie bestreitet, dass das Bild bei der Versteigerung 1969 tatsächlich versteigert worden wäre. Es sei davon auszugehen, dass das Gutachten des Hr. Friedländer - gestorben mit 91 Jahren im Jahr 1958 - bei der Versteigerung im Jahr 1969 nicht den Zustand des Gemäldes betraf, sondern dass es sich um ein kunsthistorisches Gutachten gehandelt habe, das auch auf sie, die Beklagte, als Eigentümerin Bezug genommen habe. H. habe das Gemälde nicht als öffentlich bestellter und vereidigter Versteigerer versteigert.

Die Beklagte bestreitet ferner, dass K. das Gemälde 1989 bei einer Versteigerung in New York erworben habe. Sie beruft sich auf die so genannte Washingtoner Erklärung vom 3. Dezember 1998 von einer Reihe von Staaten, u. a. der Bundesrepublik Deutschland sowie auf die hierzu ergangene so genannte Gemeinsame Erklärung der Bundesregierung, der Länder und der Kommunalen Spitzenverbände vom Dezember 1999 und die hierzu herausgegebene Handreichung zur Umsetzung dieser Gemeinsamen Erklärung. Dem Kläger fehle das Feststellungsinteresse, da sie, die Beklagte, sich vorgerichtlich nicht darauf berufen habe, Eigentümerin zu sein. Der Kläger sei aber auch tatsächlich nicht Eigentümer. Ein Eigentumserwerb sei im Hinblick auf die Bestimmungen des alliierten Rückerstattungsrechts ausgeschlossen, nämlich des US-Militärgesetzes Nr. 59, des Gesetzes Nr. 59 in der britischen Besatzungszone und der Rückerstattungsanordnung für Berlin, die unabhängig davon, dass die Anmeldefristen zur Rückerstattung abgelaufen seien, noch in Kraft seien. Die Versteigerung im Jahr 1969 sei keine öffentliche Versteigerung im Sinne des § 935 Abs. 2 BGB gewesen. Der Kläger könne sich auch nicht auf den guten Glauben eines unbekannten Dritten berufen. Den Erwerber treffe auch eine Nachforschungspflicht beim Kauf eines solchen Gemäldes. Gerade im Kunst- und Antiquitätenhandel seien insofern strenge Anforderungen zu stellen, vorliegend um so mehr, als die notwendigen Nachforschungen in kurzer Zeit hätten angestellt werden können. Der F.-Katalog errege schon deshalb Verdacht, weil ein Altmeistergemälde völlig ohne Provenienz angeboten werde.
Selbst wenn K. das Gemälde bei der Versteigerung im Jahr 1989 ersteigert hätte, habe kein gutgläubiger Erwerb vorgelegen, und zwar zum einen wegen der Nachforschungspflichten des Erwerbers, zum anderen wegen des Herkunftshinweises im Katalog.

Der Kläger habe auch keinen Anspruch auf Löschung der beanstandeten Einträge. Er sei nicht passiv legitimiert, weil der Eintrag bei "lostart.de" auf die Erbengemeinschaft D. zurückgehe. Unabhängig von der Eigentumsfrage habe sie das Recht, durch die Einträge darauf hinzuweisen, dass es sich um NS-Beutekunst handele. Darauf müsste auch jeder Auktionator hinweisen. [...]

\section{Entscheidungsgründe}

Die Klage ist zulässig, aber unbegründet.

I. Am Feststellungsinteresse des Klägers gibt es keinen Zweifel. Jedenfalls in den im Rahmen dieses Rechtsstreits ausgetauschten Schriftsätzen hat sich die Beklagte ihres Eigentums an dem streitgegenständlichen Gemälde berühmt und geltend gemacht, der Kläger sei nicht Eigentümer. Ob die vorgerichtliche Aufforderung, das Gemälde herauszugeben, daher nur "appellativen“ Charakter hatte, ist irrelevant.

II. 1. Bei der Frage, ob der Kläger Eigentümer des Bildes ist, kommt es nicht auf die Washingtoner bzw. die Gemeinsame Erklärung an, die keinen Einfluss auf die privatrechtliche Rechtspositionen außerhalb der öffentlichen Hand haben. Auch der Umstand, dass unter Geltung der zur Zeit der Besetzung Deutschlands nach dem Zweiten Weltkrieg erlassenen Rückgabegesetze ein Rückgabeanspruch der Beklagten bestanden haben mag, hat auf die heutige Rechtslage keinen Einfluss, weil nämlich ein Rückgabeantrag unstreitig nicht gestellt worden ist, dessen Bescheidung allein zu einer Rechtsänderung hätte führen können.

2. Die Beklagte war ursprünglich Eigentümerin des Gemäldes. Dies ist zwischen den Parteien unstreitig.

Es muss auch davon ausgegangen werden, dass das Gemälde der Beklagten im Sinne des § 935 Abs. 1 BGB abhanden gekommen ist. Dem Kläger ist zwar zuzugeben, dass insoweit die Darlegungs- und Beweislast bei der Beklagten liegt. Sie ist dieser Darlegungslast jedoch nachgekommen. Der Umstand, dass die Bundesrepublik Deutschland in einer Vereinbarung mit der Beklagten feststellte, dass ein ebenfalls im Jahr 1935 durch das Auktionshaus C. versteigertes Bild der Beklagten einen verfolgungsbedingten Vermögensverlust darstelle, weil die Eheleute D., hinsichtlich der Gesellschaftsanteile an der Beklagten Vermächtnisnehmer des 1929 gestorbenen N., nach 1933 aufgrund ihrer Religionszugehörigkeit so genannten rassischen Verfolgungsmaßnahmen ausgesetzt gewesen seien und sich daher gezwungen gesehen hätten, den Geschäftsbetrieb der Beklagten aufzugeben, zeigt hinreichend deutlich, dass an der Verfolgung der Eheleute D. durch die Nationalsozialisten und 
der darauf beruhenden Versteigerung des Vermögens der Beklagten kein ernsthafter Zweifel bestehen kann. Auch ist kein Grund ersichtlich oder vom Kläger aufgezeigt worden, weshalb sich die Beklagte seinerzeit in Liquidation befunden haben oder weshalb ihr Vermögen versteigert worden sein sollte, wenn nicht aus verfolgungsbedingten Gründen. Der Kläger greift dies lediglich mit verschiedenen handelsregisterlichen Dokumenten an, die belegen sollen, dass Zweifel daran bestünden, dass durch die Beschlagnahme des Vermögens der Eheleute D. die Beklagte enteignet worden wäre. Dies kann die Kammer nicht nachvollziehen. Einen anderen auch nur im Ansatz nahe liegenden Grund kann auch der Kläger nicht aufzeigen. Zudem ist es unstreitig, dass der ehemalige Inhaber der Beklagten N. sowie diejenigen, denen die Gesellschaftsanteile nach seinem Tod aufgrund seines Vermächtnisses zufallen sollten, die Eheleute D., und die Geschäftsführer des Unternehmens Juden waren. Inwiefern sich aus den vom Kläger vorgelegten Dokumenten Zweifel an einer verfolgungsbedingten Beschlagnahme des Vermögens der Beklagten ergeben sollten, ist unklar, weshalb davon auszugehen ist, dass die Verfolgung der bereits im Jahr 1933 emigrierten Eheleute D. durch die Nationalsozialisten wegen ihrer Religionszugehörigkeit der Grund für die Versteigerung war. Dann aber gilt das streitgegenständliche Bild als abhanden gekommen.

3. Ein gutgläubiger Erwerb auf der vom Kläger angeführten Versteigerung der Galerie F. im Jahr 1969 kann nicht festgestellt werden. Zwar sind die Einwendungen des Klägers gegen die grundsätzlichen Bedenken einer Einordnung einer freiwilligen Versteigerung als eine öffentliche Versteigerung im Sinne des $\S 935$ Abs. 2 BGB begründet. Die Kammer schließt sich insoweit der Rechtsprechung des Bundesgerichtshofs (NJW 1990, 899) an. Der Kläger hat aber trotz des Bestreitens durch die Beklagte nicht dargelegt, geschweige denn entsprechenden Beweis angeboten, dass das Gemälde tatsächlich auf der Versteigerung im Jahr 1969 veräußert worden wäre. Aus dem von ihm vorgelegten Katalog ergibt sich lediglich, dass es auf der Versteigerung angeboten wurde. Es ist auch durchaus nicht fernliegend, dass das Bild angeboten, aber nicht versteigert wurde, wie die Verkaufsversuche aus dem Jahr 1993 und 1997 zeigen. Dafür spricht auch, dass noch im I.-Katalog aus dem Jahr 1989 zwar noch die Beklagte für die Zeit vor 1932, nicht aber ein Erwerber aus dem Jahr 1969 und auch im L.-Katalog aus dem Jahr 1993 lediglich die Beklagte angegeben war. Auf einen gutgläubigen Erwerb im Sinne des $\S 935$ Abs. 2 BGB bei der Versteigerung im Jahr 1969 kann sich der Kläger daher nicht berufen.

4. Soweit sich der Kläger auf einen gutgläubigen Erwerb im Rahmen der Versteigerung im Jahr 1989 beruft, ist unstreitig, dass das Bild dort zur Versteigerung aufgerufen worden ist. $\mathrm{Ob}$ aufgrund der dortigen Versteigerung Eigentum erworben werden konnte, richtet sich aber nach dem Recht der belegenen Sache gemäß Art. 43 EGBGB.

a. Sofern das Bild nicht körperlich nach New York gelangt war, was allerdings eher fernliegend erscheint, galt deutsches Recht. Ein gutgläubiger Erwerb durch die Versteigerung in New York im Sinne des § 935 Abs. 2 BGB kam dann nicht in Betracht, da der dortige Versteigerer jedenfalls kein öffentlich bestellter und vereidigter Versteigerer war.

b. Sofern das Bild sich aber körperlich in New York befand, galt für die Frage eines gutgläubigen Erwerbs aber das Recht des amerikanischen Bundesstaats New York. Nach der Entscheidung Menzel vs. List N. Y. S. 2d 804 (N.Y. Sup. Ct. 1966) kam aber ein gutgläubiger Erwerb des Gemäldes nicht in Betracht. Dies ist zwischen den Parteien auch unstreitig.

5. Der Kläger kann sich auch nicht mit Erfolg auf eine Ersitzung des Bildes berufen. Dafür ist nämlich erforderlich, dass der Besitzer nicht bösgläubig war. Der Kläger beruft sich insofern nach § 943 BGB auf die Zeit, in der sich das Bild im Besitz seines Schwiegervaters, K., befunden habe. Zwar ist es grundsätzlich Sache der Beklagten darzulegen und zu beweisen, dass sich der Kläger bzw. der Vorbesitzer, sein Schwiegervater, nicht in gutem Glauben hinsichtlich ihres Eigenbesitzes befunden haben. Die Beklagte hat aber Anhaltspunkte vorgebracht, die einen guten Glauben äußerst zweifelhaft erscheinen lassen. Der Vorbesitzer K. hat im Jahr 1993 versucht, das Bild über L. zu veräußern, was ihm nicht gelungen ist. Im dortigen Versteigerungskatalog hieß es, dass der Voreigentümer die Beklagte sei. Der Kläger trägt mit keinem Wort vor, woran die seinerzeitige Versteigerung gescheitert ist und ob er hierüber mit dem die Versteigerung organisierenden Auktionshaus gesprochen hat. Es erscheint mehr als nahe liegend, dass der Verkauf des Bildes an eben dem Hinweis auf den Voreigentümer gescheitert ist.

Auch im Hinblick auf die versuchte Veräußerung in Spanien im Jahr 1997 verliert der Kläger kein Wort, weshalb dieser Veräußerungsversuch gescheitert ist. Dies wäre aber notwendig gewesen. Denn trotz der Darlegungs- und Beweislastverteilung im Rahmen des § 937 BGB obliegt es auch dem Kläger, sich über die entscheidungserheblichen Tatsachen nicht nur wahrheitsgemäß, sondern auch vollständig zu erklären (§ 138 Abs. 1 ZPO). Dabei kommt vorliegend insbesondere zum Tragen, dass die Beklagte außerhalb des insoweit erheblichen Geschehensablaufs steht und keine nähere Kenntnis der maßgebenden Tatsachen besitzen kann. Dies führt zu einer erweiterten Darlegungslast des Klägers, der er nicht nachgekommen ist. Da somit weder hinsichtlich des K. noch hinsichtlich des Klägers angenommen werden kann, dass die 10-jährige Ersitzungszeit in Eigenbesitz des Bildes verstrichen ist, konnte dadurch kein Eigentum entstehen.

Soweit sich der Kläger auf eine Verjährung des Herausgabeanspruchs beruft, ist dies nicht von Belang, da auch die eingetretene Verjährung den Bestand des Anspruchs unberührt lässt und lediglich dessen gerichtliche Durchsetzbarkeit entfallen lässt.

III. Da der Kläger schon sein Eigentum an dem Gemälde nicht darlegen kann, fehlt es schon aus diesem Grund an einem Anspruch auf Löschung der streitgegenständlichen Eintragungen in den Registern „artloss.com" und „lostart.de“, da nämlich nicht ersichtlich ist, in welcher Weise dadurch Rechte des Klägers beeinträchtigt sein könnten. [...] 